\title{
Retour d'expérience des transferts en HéliSmur de patients Covid-19
}

\section{Feedback from Helicopter Emergency Medical Service for Transport of COVID-19 Patients}

\author{
C. Chollet-Xémard · D. Michel · P. Szuster - D. Cervellin · E. Lecarpentier \\ Reçu le 6 juillet 2020; accepté le 4 août 2020 \\ (C) SFMU et Lavoisier SAS 2020
}

Résumé L'augmentation du nombre d'hospitalisations en réanimation de patients graves atteints de la Covid-19 a nécessité le transfert d'un certain nombre d'entre eux vers des régions moins touchées que le Grand Est et l'Île-de-France afin de ne pas dégrader la qualité des soins. Les HéliSmur ont fait partie intégrante du dispositif d'évacuation de ces patients. Utilisés au quotidien, ils ont confirmé leur utilisation en cas de crise où la problématique des élongations est une difficulté. Cependant, le recours aux HéliSmur a nécessité une adaptation de tous à de nouvelles modalités opérationnelles. Le transport de patients critiques, le port d'un équipement de protection individuelle par l'équipe médicale et les membres d'équipage ainsi que les procédures renforcées de bionettoyage ont impacté les temps d'intervention mais aussi la charge mentale des personnes à bord. La mise en place d'équipes médicales dédiées et rompues aux transferts héliportés a permis d'optimiser la prise en charge complexe de ces patients tant sur le plan médical qu'aéronautique. Nous présentons notre retour d'expérience des transferts en HéliSmur que nous avons réalisés au départ de la région francilienne.

Mots clés Covid-19 · Transfert · HéliSmur patients. Used on a daily basis, they have confirmed their use in the event of a crisis where the problem of elongations is difficulty. However, the use of Helicopter Emergency Medical Service requires everyone to adapt to new operational methods. The transport of critical patients, the wearing of personal protective equipment by the medical team and crew members as well as the reinforced bio-cleaning procedures have impacted the intervention times but also the mental load of the people on board. The establishment of dedicated medical teams experienced in helicopter transfers has made it possible to optimize the complex care of these patients, both medically and aeronautically. We present our feedback from the Helicopter Emergency Medical Service transfers that we carried out from the Ile-de-France region.

Keywords COVID-19 - Transfer · Helicopter Emergency Medical Service

\section{Introduction}

Les transferts interrégionaux et internationaux organisés dans le cadre de l'épidémie de la Covid-19 ont nécessité la mobilisation de nombreux vecteurs aériens, terrestres ou maritimes. Utilisés au quotidien dans le cadre de l'aide médicale urgente par les Smur [1], les hélicoptères sanitaires ont été largement sollicités dans ce contexte et ont fait partie intégrante du dispositif. Les distances à parcourir et le nombre de patients à transférer ont, pour les Samu, logiquement nécessité le recours aux HéliSmur afin d'optimiser les temps d'intervention des équipes médicales. Le recours aux HéliSmur pendant la crise sanitaire a nécessité une adaptation de tous à de nouvelles modalités opérationnelles (Fig. 1A,B). Le transport de patients critiques, la protection des personnels, le bionettoyage de l'hélicoptère et du matériel embarqué ont impacté les prises en charge, augmentant le temps d'intervention et la charge mentale pour les personnes à bord. 


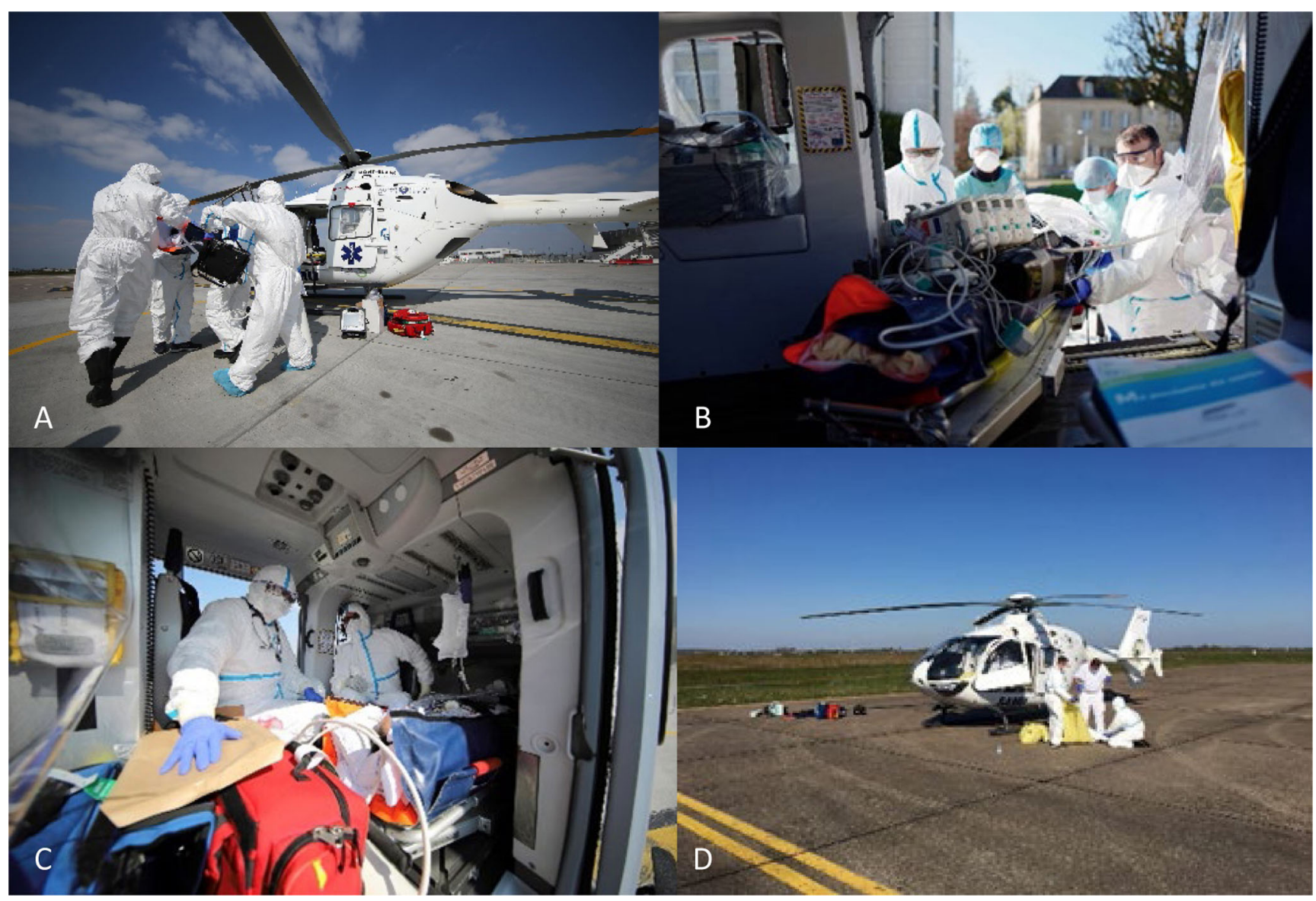

Fig. 1 A et B : Embarquement d'un patient par une équipe Smur en tenue de protection dans l'hélicoptère H135 Samu Ile-de-France (Airbus Helicopters). C : Cellule sanitaire du H 135 Samu Ile-de-France (Airbus Helicopters) : aperçu des contraintes environnementales et logistiques D : Application de la procédure de déshabillage en binôme sur l'aéroport de Tours devant le H135 Samu Ile-de-France (Airbus Helicopters)

\section{Transport de patients critiques}

La réalisation de gestes techniques en vol, a fortiori, chez des patients critiques à haut risque de contamination biologique est très difficile (Fig. 1C). La gravité et l'instabilité de ces patients nécessitent, à la prise en charge, une réévaluation clinique par le médecin du Smur. Elle permet de s'assurer que l'état clinique du patient est compatible avec un transfert héliporté (Tableau 1) en tenant compte des contraintes environnementales propres au type d'hélicoptère comme la taille

Tableau 1 Critères cliniques retenus pour les transferts héliportés de patients atteints de la Covid-19

Fraction inspirée d'oxygène $\leq 50 \%$

Pression expiratoire positive $\leq 10 \mathrm{cmH}_{2} \mathrm{O}$

Débit de noradrénaline $\leq 0,15 \mu \mathrm{g} . \mathrm{kg}-1$.min-1

Poids corporel $<100 \mathrm{~kg}$ de la cabine sanitaire, de la civière, et le poids du patient (Tableau 2).

L'équipe médicale doit s'assurer d'une mise en condition adaptée afin de limiter le risque d'exposition virale [2] :

- existence d'un système clos d'aspiration ;

- présence d'un filtre hydrophobe à membrane plissée à l'extrémité de la branche expiratoire du circuit patient afin de protéger le ventilateur, mais aussi d'un filtre échangeur de chaleur et d'humidité (HME) positionné sur la pièce en Y.

Le changement de ventilateur entraîne une rupture d'étanchéité du circuit. Cette manœuvre est à haut risque d'une part de désaturation entrainée par le dérecrutement lié à la perte de pression et, d'autre part, d'exposition virale. Elle doit être réalisée après une pause expiratoire.

Les fins de perfusions, notamment pour la sédation continue et les catécholamines, sont également à anticiper particulièrement en cas de relais terrestre pouvant allonger les durées de transfert. L'existence d'une curarisation nécessite 
Tableau 2 Caractéristiques techniques de l'hélicoptère H135 Samu Île-de-France (Airbus Helicopters) et de l'hélicoptère H145 (Airbus Helicopters)

\begin{tabular}{|lll|}
\hline Caractéristiques techniques & H135 Samu Île-de-France & H145 \\
\hline Taille de la civière $(\mathrm{cm})$ & $46 \times 186$ & En fonction du kit patient \\
Largeur de la cabine $(\mathrm{cm})$ & 123 & 140 \\
Longueur de la cabine $(\mathrm{cm})$ & 276 & 357 \\
Hauteur de la cabine au niveau patient $(\mathrm{cm})$ & 115 & 122 \\
Volume de la cabine sanitaire $\left(\mathrm{m}^{3}\right)$ & 5,04 & 6,03 \\
Poids maximum supporté par le système de fixation de la civière $(\mathrm{kg})$ & 150 & 150 \\
\hline
\end{tabular}

une immobilisation de la tête du patient, qui peut être réalisée par un collier cervical ou plus simplement par le matelas à dépression. Dans ce dernier cas, il est préférable de positionner la valve à dépression au niveau de la tête du patient et non au niveau des pieds qui ne seront plus accessibles pendant le vol.

La visibilité des éléments de surveillance des paramètres vitaux lors du vol est essentielle ainsi que l'accessibilité des seringues autopousseuses, du ventilateur et du multiparamètre. Les vibrations émises par les hélicoptères en vol peuvent interférer avec les éléments de surveillance, ce qui requiert une surveillance particulièrement rapprochée de ces patients par l'équipe médicale.

\section{Protection des personnels}

Le recours à des équipements de protection individuelle (EPI) pour les équipes médicales mais aussi pour les membres d'équipage a été incontournable dans ce contexte et a nécessité une grande adaptabilité de tous. L'EPI comprenait une combinaison de type Tyvek ${ }^{\circledR}$ (DuPont, Wilmington, États-Unis), des surchaussures, une paire de lunettes, un masque FFP2 et une double paire de gants. Les procédures de prise en charge ont été ajustées :

- seul l'assistant de vol, équipé d'une double paire de gants, aidait l'équipe médicale à l'installation du patient à bord de l'hélicoptère, il enlevait ensuite la paire de gants extérieure avant d'accéder au poste de pilotage ;

- retrait des mousses des microphones afin de faciliter le bionettoyage ;

- pose d'une paroi souple permettant d'isoler le cockpit de la cabine sanitaire ;

- obturation des buses de ventilation de la cabine et verrouillage général de la ventilation.

Les procédures d'habillage/déshabillage en binôme doivent être maîtrisées par les équipes médicales et les membres d'équipage afin d'éviter une contamination secondaire (Fig. 1D).
L'utilisation de housse de transport pour victime contaminée, de type sarcophage, offre l'avantage d'isoler complètement le patient, limitant considérablement le risque de contamination. Cependant, elle rend inaccessible le patient pendant le transfert, situation hautement risquée pour ces patients intubés, ventilés, sédatés et souvent sous catécholamines. Un autre système a été développé pendant la crise sanitaire, améliorant l'accessibilité du patient durant le vol. Il s'agit d'une bulle de réanimation aéroportée pour virus émergents (BRAVE) inventée par une entreprise dijonnaise (Bâche 21, Dijon, France) avec l'aide du Samu de Dijon et des ingénieurs d'Airbus Helicopters (Fig. 2). Actuellement, une vingtaine d'HéliSmur en sont équipés. La dotation d'une BRAVE pour chaque HéliSmur devrait être effective prochainement.

\section{Bionettoyage}

Il a nécessité l'adaptation des procédures habituelles pour ces transports. Ainsi dès lors que l'équipe médicale et le patient étaient sortis de l'hélicoptère, la totalité des surfaces de la cellule sanitaire, du matériel fixe et les casques était bionettoyée à l'aide de lingettes imbibées de produit revendiquant la norme virucidie (EN 14476). Ce bionettoyage était réalisé par l'assistant de vol, avec son EPI, lors du

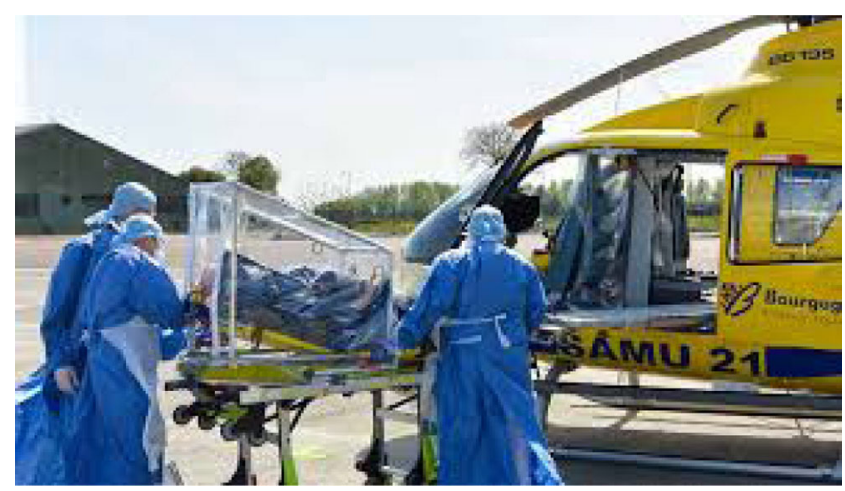

Fig. 2 Bulle sanitaire aéroportée pour virus émergents (BRAVE) (Bâche 21, Dijon, France) 
transfert du patient de l'hélicoptère sanitaire vers le service receveur. Cette organisation a permis d'optimiser les durées d'intervention Smur, permettant la réalisation de deux rotations Paris-Province journalières pour des destinations situées entre 1 heure et 1 heure 30 de vol. Elle a permis également de limiter le temps d'exposition des personnes à bord, le vol retour pouvait ainsi s'effectuer dans des conditions plus confortables pour les membres d'équipage et l'équipe médicale qui n'avaient plus obligation de porter leurs EPI.

\section{Discussion}

Dès le début de la crise, la mise en place d'équipes médicales dédiées et rompues aux transports héliportés a facilité la prise en charge complexe de ces patients tant sur le plan médical qu'aéronautique. Cela a permis d'accélérer l'acquisition de l'expertise de ces transferts critiques intégrant sévérité des patients, contraintes environnementales liées à l'hélicoptère et risque biologique. Les temps de prise en charge, de ce fait, ont été largement diminués, permettant plusieurs rotations journalières.

Indispensables de par leur rapidité de mise en œuvre, complémentaires aux dispositifs d'évacuation de masse de patients par voie terrestre, les HéliSmur ont assuré, durant toute la crise, des transferts de patients Covid-19. Ils ont fait partie intégrante des transports intrarégionaux, interrégionaux, internationaux et ont largement contribué à sauver des vies [3]. Utilisés au quotidien dans le cadre de l'aide médicale urgente, les HéliSmur ont été d'une incroyable efficacité dans le cadre de cette crise sanitaire, malgré des contraintes nouvelles. Une étroite collaboration entre équipes médicales, membres d'équipage et opérateurs a permis une adaptation rapide et efficace des modalités opérationnelles de ces transferts.

Toutefois, des pistes d'amélioration sont envisageables :

- gabarit de l'hélicoptère et volume intérieur de la cellule sanitaire : ils peuvent varier d'un hélicoptère à l'autre en fonction du type de machine utilisée. Par exemple, le volume intérieur de la cellule sanitaire d'un hélicoptère H145 (Airbus Helicopters) est plus important que celui d'un hélicoptère H135 (Airbus Helicopters) offrant à l'équipe médicale davantage d'espace facilitant ainsi la réalisation de gestes durant le vol (Fig. 3AB). De plus, en fonction de l'aménagement intérieur, l'hélicoptère H145 offre la possibilité d'embarquer une troisième personne de l'équipe médicale (Fig. 3C), point essentiel pour les personnels en formation. En effet, ces prises en charge très spécifiques soumises à de fortes contraintes environnementales (installation du patient et du matériel à bord, anticipation d'une dégradation du patient, pertinence d'un poser en urgence, contraintes du vol, bénéfice/risque d'un transport héliporté...) doivent être maîtrisées par les équipes médicales embarquées. Aujourd'hui, l'ensemble des bases HéliSmur accueillant des personnels en formation devraient pouvoir bénéficier d'hélicoptères dont le gabarit permet d'embarquer trois personnes de l'équipe médicale. En fonction de l'aménagement intérieur, il est également possible d'embarquer deux patients, ce qui peut s'avérer utile en cas d'afflux massif de patients à transférer (Fig. 3D). Néanmoins, cette possibilité doit rester modulable, car elle réduit l'espace de la cellule sanitaire; de plus, sa mise en œuvre doit être rapide afin de ne pas impacter les délais d'intervention. Une amélioration des connaissances des contraintes techniques liées à chaque appareil (Tableau 2) est nécessaire pour les médecins posant l'indication de ce type de vecteur, et ce afin de rationaliser son utilisation. Ces contraintes techniques sont disponibles dans le manuel d'exploitation (MANEX) de chaque hélicoptère. Le rapport poids/taille peut avoir toute son importance, la largeur de la civière n'excédant pas $46 \mathrm{~cm}$ pour l'hélicoptère H135. À titre d'exemple, le poids d'une patiente, d'une part, avait été sous-évalué par les réanimateurs et, d'autre part, sa taille n'avait pas était prise en compte. De ce fait, la morphologie de cette patiente de petite taille n'a pas permis son installation sur la civière de l'hélicoptère. La mobilisation inutile de l'HéliSmur dans ce contexte, ainsi que la réorganisation de l'évacuation sanitaire de la patiente par un autre moyen (vecteur terrestre puis évacuation par hélicoptère Caracal de l'armée), n'a pas permis la double rotation journalière de notre équipe prévue dans le cadre du hub d'Orly ;

- accessibilité des hôpitaux : En 2008, l'AFHSH recommandait de rendre obligatoire la création d'une aire de poser pour toute structure accueillant ou exportant des patients en état critique [4]. Aujourd'hui encore, de trop nombreux établissements de soins s'octroient la possibilité de fermer leur zone de poser ou en sont dépourvus, jugeant cet équipement, à fort coût financier, non prioritaire. De ce fait, les relais terrestres sont de plus en plus nombreux et impactent fortement le gain de temps et la disponibilité des HéliSmur. Le relais terrestre effectué par une équipe de Smur territorialement compétente sur l'aire de poser optimiserait ainsi la disponibilité de l'HéliSmur. Cela n'a pas toujours été réalisable dans le cadre des évacuations héliportées, limitant le nombre de rotations journalières. Enfin, la multiplication des brancardages et le transport par voie terrestre, parfois effectué en ambulance privée, restent délicats et risqués pour ces patients fragiles présentant des syndromes de détresse respiratoire aiguë (SDRA) à SARS-Cov-2. Aucun décès durant ces évacuations par HéliSmur n'a cependant été rapporté à notre connaissance. Nous n'avons pas d'évaluation du risque du transport héliporté en termes de surmortalité comparé 


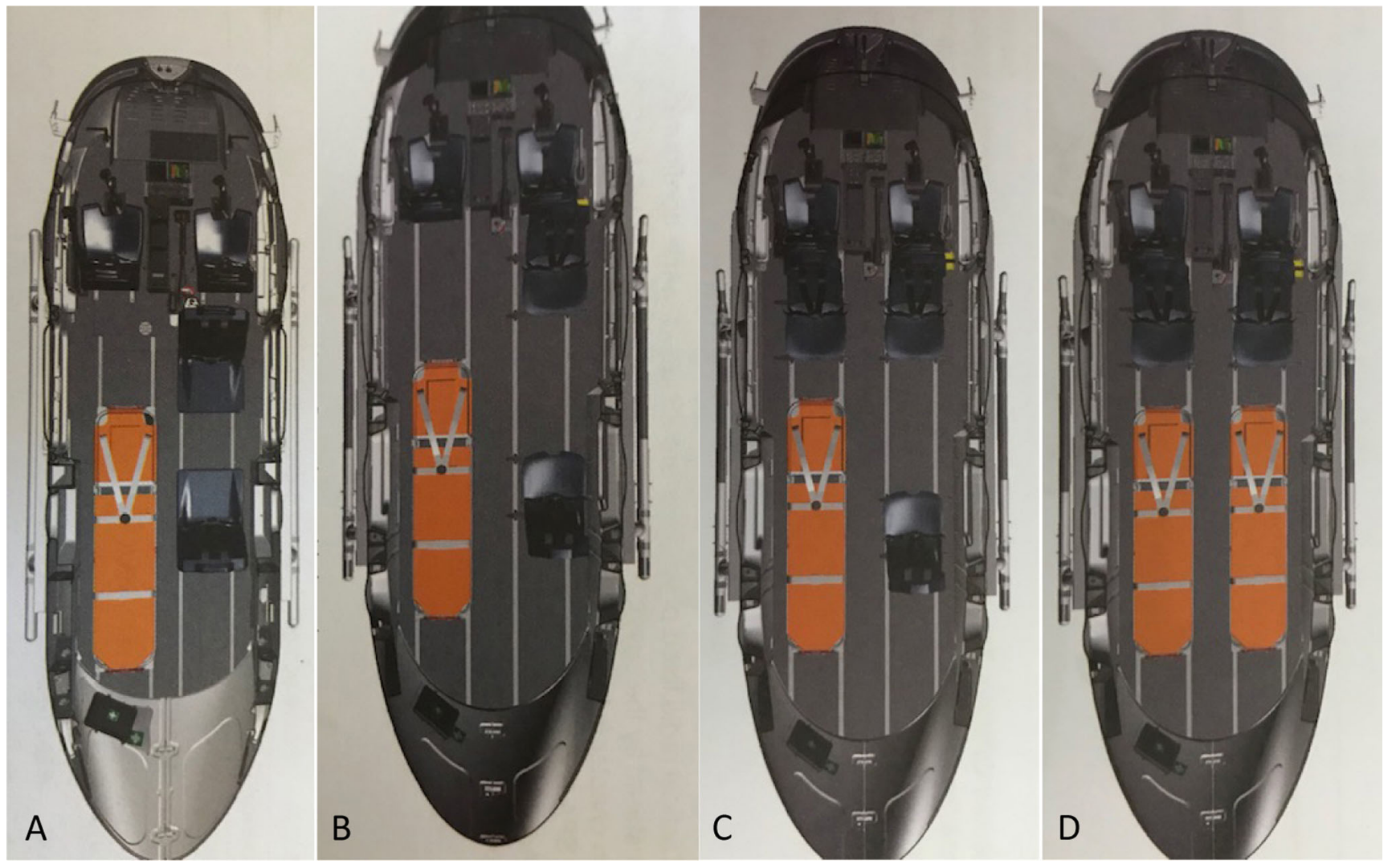

Fig. 3 A : Cellule sanitaire d'un hélicoptère H135 (Airbus Helicopters). B : Cellule sanitaire d'un hélicoptère H145 (Airbus Helicopters). C : Aménagement d'un H145 (Airbus Helicopters) pour une équipe médicale composée de 3 personnes. D : Aménagement d'un H145 (Airbus Helicopters) pour un transport de 2 patients

à d'autres moyens de transport que ce soit par voie terrestre, maritime, aérienne ou ferroviaire ni par rapport au fait de ne pas transférer les patients. Des études cliniques sont nécessaires.

La mobilisation précoce des HéliSmur armés par des équipes médicales dédiées semble incontournable de par leur rapidité de mise en œuvre. Notre opérateur a mis à disposition dans le cadre du hub d'Orly un deuxième hélicoptère, augmentant la capacité d'évacuation de quatre patients par jour pour notre Samu [5]. Cette montée en puissance, de décision probablement plus facile et rapide que la mobilisation d'aéronefs de l'armée, pourrait être envisageable dès les premières évacuations nécessaires. De plus, les HéliSmur peuvent se poser directement dans un établissement de soins dès lors qu'il dispose d'une aire de poser, limitant ainsi les ruptures de charge, ce qui n'est pas toujours le cas pour des hélicoptères dont le gabarit et le poids sont plus importants (Caracal, Caïman).

Les HéliSmur interviennent au quotidien dans le cadre de l'AMU pour les transferts d'enfants ou de nourrissons vers la province. Ils auraient encore une fois toute leur place pour le transfert d'un grand nombre de patients pédiatriques.
La problématique de la housse de transport pour victime contaminée par un virus de type Ebola dans un hélicoptère sanitaire reste entière. L'installation dans la housse de transport d'un patient sous catécholamines, intubé/ventilé sous sédation continue est extrêmement compliquée et délicate afin d'éviter tout arrachage des abords veineux ou extubation. Si un transfert dans ces conditions devait s'envisager, il ne pourrait pas se réaliser dans un hélicoptère H135 trop exigu. Les critères à la fois cliniques et de mise en condition des patients devraient être très stricts et ne pas concerner forcément les patients intubés/ventilés. Pour ces derniers, en l'absence de housse de transport, la protection des personnels et des membres d'équipage revêtus d'EPI adaptés reste l'alternative associée aux mesures déjà mises en place durant la crise Covid-19.

\section{Conclusion}

La crise sanitaire à laquelle nous avons fait face a mis en lumière la formidable force de frappe des Samu et des Smur que ce soit en début janvier lors des rapatriements des ressortissants français de Wuhan, en régulation médicale ou sur 
le terrain. Cette crise s'est inscrite dans la durée, et les Samu et les Smur ont su proposer et organiser des dispositifs innovants comme les transferts par voie ferroviaire, maritime ou aérienne. Utilisés au quotidien dans le cadre de l'AMU, les HéliSmur ont fait preuve d'un incroyable réactivité dans le transfert de ces patients atteints de la Covid-19 et les opérateurs, d'une grande agilité. Les modalités opérationnelles de ces transferts ont dû être modifiées, nécessitant une grande adaptabilité des équipes médicales et des membres d'équi- page. La force et l'abnégation de tous auront permis le transport de 455 patients en HéliSmur que ce soit sur le territoire national ou à l'international.

Liens d'intérêts : P. Szuster et D. Cervellin sont pilotes chez Mont Blanc Hélicoptères. L'hélicoptère H135 Samu Îlede-France est opéré par Mont Blanc Hélicoptères. Il est basé à l'hôpital universitaire Henri-Mondor, utilisé et coordonné par le Samu 94.

\section{Éléments remarquables}

- Mise en place d'équipes médicales Smur dédiées et rompues aux transports héliportés

- Adaptation rapide des équipes médicales et des membres d'équipage aux nouvelles modalités opérationnelles de ces transferts

- Réactivité des HéliSmur, agilité des opérateurs

\section{Pistes d'amélioration}

- Équiper l'ensemble des bases HéliSmur d'hélicoptère ayant un gabarit de type H145

- Accessibilité des hôpitaux : création d'aire de poser, interdiction de fermer une zone de poser sans autorisation préalable

- Améliorer la connaissance des contraintes environnementales de l'hélicoptère par les prescripteurs

\section{Références}

1. Samu et Urgence de France (2017) Doctrine d'emploi des hélicoptères dans le cadre de l'aide médicale urgente. www.samu-urgences-de-france.fr (Dernier accès le 24 juillet 2020)

2. Société de réanimation de langue française (SRLF) (2020) Préconisations ventilation - gestion du circuit d'un ventilateur de réanimation. www.srlf.org (Dernier accès le 24 juillet 2020)

3. Morin F, Peschanski N, Ricard Hibon A, et al (2020) Retour d'expérience sur les transports Smur des patients Covid-19. Ann Fr Med Urgence 10:314-20
4. Letellier N (2008) Le livre blanc de l'hélicoptère sanitaire. AFHSH, Paris

5. Lecarpentier E, Travers N, Jbeili C, Chollet-Xémard C (2020) Retour d'expérience sur le centre médical de coordination et d'évacuation aérien Covid - Paris-Orly. Ann Fr Med Urgence 10:278-87 